\title{
Recognizing Rectangle of Influence Drawable Graphs * (extended abstract)
}

\author{
H. ElGindy ${ }^{1}$, G. Liotta ${ }^{2}$, A. Lubiw ${ }^{3}$, H. Meijer ${ }^{4}$, S. H. Whitesides ${ }^{5}$
}

1 Department of Computer Science, The University of Newcastle, Callaghan NSW 2308, Australia.

hossamecs.newcastle.edu.au

2 Dipartimento di Informatica e Sistemistica, Università di Roma 'La Sapienza', Roma, Italia.

liottalinfokit.dis. uniroma1.it

3 Department of Computer Science, University of Waterloo, Waterloo Ontario N2L 3G1 Canada.

alubiw@math .uwaterloo.ca

4 Computing and Information Science Department, Queen's University, Kingston, Ontario K7L 3N6 Canada. henkequcis.queensu.ca

${ }^{5}$ School of Computer Science, McGill University, Montréal, Québec, H3A 2A7 Canada. sueecs.mcgill.ca

\begin{abstract}
Given two points $x$ and $y$ in the plane the rectangle of influence of $x$ and $y$ is the axis-aligned rectangle having $x$ and $y$ at opposite corners. The rectangle of influence drawing of a graph $G$ is a straight-line drawing of $G$ such that (i) for each pair of adjacent vertices $u, v$ of $G$ the rectangle of influence of the points representing $u$ and $v$ is empty (i.e. does not contain any other point representing any other vertex of $G$ except possibly the two representing $u$ and $v$ ) and (ii) for each pair of non adjacent vertices $u, v$ of $G$ the rectangle of influence of the points representing $u$ and $v$ is not empty. In this paper we consider several classes of graphs, and we characterize, for each class, which graphs of the class have a rectangle of influence drawing. For each class we show that the problem of testing whether a graph $G$ has a rectangle of influence drawing can be done in linear time. Furthermore, if the test for $G$ is affirmative, a rectangle of influence drawing of $G$ can be constructed in linear time with the real RAM model of computation.
\end{abstract}

\section{Introduction and Overview}

The problem of drawing a graph in the plane has been receiving increasing attention due to the large number of applications, including VLSI layout, algorithm

\footnotetext{
* Research supported in part by the Progetto Finalizzato Sistemi Informatici e Calcolo Parallelo and the grant 94.00023.CT07 of the Italian National Research Council, and by the Esprit II BRA of the European Community (project ALCOM).
} 
animation, visual languages and CASE tools. Vertices are usually represented as points in the plane and edges as simple Jordan curves connecting pairs of points. Graph drawing tools and algorithms usually adopt a given drawing convention (for example edges are represented by straight-line segments or orthogonal chains) and attempt to solve several optimization problems (e.g. minimizing the area of the drawing, maximizing the number of convex faces, minimizing the number of bends along the edges etc.). For an extensive overview of graph drawing problems and algorithms the reader is referred to the bibliography by Di Battista, Eades, Tamassia and Tollis [6].

A straight-line drawing (for example, see $[11,12,15]$ ) of a graph $G$ is a mapping of each vertex $v$ of $G$ to a distinct point of the plane and of each edge $(u, v)$ of $G$ to a straight-line segment connecting the points that represent $u$ and $v$. Given a pair $u, v$ of points in the plane, a proximity region of $u$ and $v$ is a suitably defined portion of the plane (e.g. the smallest disk containing $u$ and $v$ ) determined by $u$ and $v$. Many definitions of the proximity region of $u$ and $v$ have been proposed in the literature. Widely used definitions include: (i) closed and open disks (also called Gabriel regions [19] and modified Gabriel regions [4], respectively) having $u, v$ as antipodal points; (ii) closed and open lunes (also called relatively closest regions [17], and relative neighborhood regions [24], respectively), obtained as the intersection of the two disks with centers $u$ and $v$ and radius the distance $d(u, v)$; and (iii) closed and open axis-aligned rectangles (also called closed rectangles of influence and open rectangles of influence [13]) having $u, v$ at opposite corners.

A proximity drawing of a graph $G$ is a straight-line drawing of $G$ such that (i) for each pair of adjacent vertices $u, v$ of $G$ the proximity region of the points representing $u$ and $v$ is empty (i.e. does not contain any other point representing any other vertex of $G$ except possibly the two representing $u$ and $v$ ) and (ii) for each pair of non-adjacent vertices $u, v$ of $G$ the proximity region of the points representing $u$ and $v$ is not empty. An open proximıty drawing is a proximity drawing where all proximity regions are open. Analogously, a closed proximity drawing is a proximity drawing where all proximity regions are closed.

There are two observation to make here. First, different definitions of proximity regions can give rise to different proximity drawings of the same graph $G$. Consider as a graph $G$ a simple path consisting of three vertices. It is easy to see that $G$ has a proximity drawing where the proximity region is the Gabriel region and such that the straight-line segments representing the edges of $G$ form an angle of $\pi / 2$. Consider now the problem of constructing a proximity drawing of $G$ adopting the modified Gabriel region as a proximity region. Clearly, $G$ admits such a proximity drawing, but the angle between the straight-line segments representing the edges of $G$ has to be greater than $\pi / 2$. Second, observe that the same graph may or may not have a proximity drawing depending on the choice of the proximity region. For example, a tree consisting of a vertex of degree 5 and five vertices of degree 1 has a proximity drawing where the proximity region is either the open or the closed lune [2]. However, it is easy to see that the same graph does not admit a proximity drawing where the proximity region is the Gabriel region. 
Proximity drawings have been intensively studied in recent years because they arise in many areas as descriptors of the shape or skeleton of a set of points (for example, see [16], [23]). Examples of such areas include pattern recognition and classification, geographic variation analysis, geographic information systems, computational geometry, computational morphology, and computer vision. For a complete survey on the different types of proximity regions, corresponding skeletons and their application areas, the reader is referred to the survey paper by Jaromczyk and Toussaint [14].

While techniques have been designed for the efficient computation of the skeleton of a given set of points, the problem of determining which graphs have proximity drawings has only just begun to be studied. The proximity drawability testing problem is to determine, for a given definition of proximity region, whether a graph admits a proximity drawing. Recent results in this new area of research include the following. In [18] it is proved that all biconnected outerplanar graphs have proximity drawings with the relative neighborhood proximity region and that all maximal outerplanar graphs have proximity drawings with the Gabriel proximity region. In [2] a complete characterization is given of those trees that admit a proximity drawing, for each of the following types of proximity region: (i) relative neighborhood region, (ii) relatively closest region (iii) modified Gabriel region, and (iv) Gabriel region. In [3], the authors studied the proximity drawability testing problem for trees for an infinite family of parametrized proximity regions, called $\beta$-regions, that include open and closed disks and lunes as special cases. Results that are closely related to the proximity drawability testing problem concern the drawability of trees as minimum spanning trees [10], of triangulations as delaunay triangulations [9],[8], and of planar graphs as nearest neighbor graphs [21]. A survey on the proximity drawability testing problem is in [7].

In this paper, we study the proximity drawability testing problem for several classes of graphs both in the case that the proximity region is the open rectangle of influence and in the case that the proximity region is the closed rectangle of influence. We say that a graph is an open rectangle of influence drawable graph if it admits a proximity drawing where the region of influence is the open rectangle of influence. Similarly, a graph is a closed rectangle of influence drawable graph if it admits a proximity drawing where the proximity region is the open rectangle of influence.

We briefly sketch the results presented in this paper.

- Open rectangle of influence drawable graphs.

1. Cycles and Wheels: Every wheel is open rectangle of influence drawable. No cycle consisting of more than three vertices is open rectangle of influence drawable.

2. Triangle free graphs: A triangle free graph is open rectangle of influence drawable iff it is a path.

3. Outerplanar graphs: A biconnected outerplanar graph is open rectangle of influence drawable iff it is maximal and its dual is a path. 
- Closed rectangle of influence drawable graphs.

1. Cycles and Wheels: Every cycle and every wheel is closed rectangle of influence drawable.

2. Trees: A tree is closed rectangle of influence drawable iff it either has exactly one vertex of degree four and all other vertices of degree 2 or has no degree 4 vertices and at most two degree 3 vertices.

3. Maximal outerplanar graphs: We give here a partial characterization. Any maximal outerplanar graph whose dual has at most one vertex of degree 3 is closed rectangle of influence drawable. No maximal outerplanar graph whose dual has at least three degree 3 vertices is closed rectangle of influence drawable.

Furthermore, we show that if a planar graph $G$ is open rectangle of influence drawable and there is a proximity drawing of $G$ with proximity region the open rectangle of influence such that no two edges cross, then $G$ has a planar embedding where all internal faces are 3-cycles.

A consequence of the above characterizations is that, given one of the classes of graphs considered in this paper, the test to determine whether a graph $G$ of the class is an open (closed) rectangle of influence drawable graph can be made in $O(n)$ time, where $n$ is the number of vertices of $G$. Furthermore, if the test for $G$ is affirmative, a proximity drawing of $G$ can be constructed in $O(n)$ time with the real RAM model of computation.

Besides graph drawing applications, our research is motivated by questions about rectangular visibility between points (for example, see [20], [5]). Given a set of distinct points in the plane, two points of the set are said to be rectangularly visible if their rectangle of influence is empty. Much attention has been given to rectangular visibility over the past years because of its importance in several computational geometry problems (for example the enclosure problem of $n$ points in the plane, the problem of finding the shortest Manhattan path among planar obstacles, and art gallery problems). Since the edges of a rectangle of influence drawing relate pairs of points that are rectangularly visible, the results of the present paper answer the question of recognizing, for several classes of graphs, which graphs can describe rectangular visibility relations between points in the plane.

The paper is organized as follows. Basic definitions are in Section 2. Properties of both open and closed rectangle of influence drawable graphs are in Section 3. Ciasses of open rectangle of influence drawable graphs and closed rectangle of influence drawable graphs are studied in Sections 4 and 5, respectively. Finally open problems are discussed in Section 6 . Omitted proofs can be found in the forthcoming full paper.

\section{Preliminaries}

We assume familiarity with the basic graph theoretic and geometric terminology (see also [1] and [22]). 
Given two distinct points $u, v$ of the plane, $R(u, v)$ denotes the open rectangle of influence of $u, v$, i.e. the axis-aligned open rectangle having $u$ and $v$ at opposite corners. Similarly, $R[u, v]$ denotes the closed rectangle of influence of $u, v$, i.e. the axis-aligned closed rectangle having $u$ and $v$ at opposite corners. Note that if $u$ and $v$ determine a horizontal or vertical line, then $R(u, v)$ and $R[u, v]$ become line segments which are still regarded as rectangles. We make one exception to the general definition of an open rectangle of influence: if $u, v, w$ are points on the same horizontal (vertical) line, in that order, then we assume that $R(u, w)$ contains $v$.

An open (closed) rectangle of influence drawing $\Gamma$ of a graph $G$ is a proximity drawing of $G$ where the proximity region is an open (closed) rectangle of influence. Points of $T$ representing vertices of $G$ are called vertex-points; line-segments of $\Gamma$ representing edges of $G$ are called edge-segments. If $G$ is a simple path, we make the assumption that an open rectangle of influence drawing of $G$ can have all the vertex-points lying either on an horizontal line or on a vertical line. In the rest of the paper, when it does not give rise to ambiguities, we will use the same label to denote both a vertex of $G$ and the corresponding vertex-point in $\Gamma$. Analogously, $(u, v)$ is both an edge of $G$ and the corresponding edge-segment in $\Gamma$. An open (closed) rectangle of influence drawable graph is a graph that has an open (closed) rectangle of influence drawing. In the following, open and closed rectangle of influence drawable graphs are also referred to as open RID graphs and closed RID graphs, respectively.

Let $P$ denote a set of distinct points in the plane. We now define several graphs having vertex-point set $P$. The open rectangle of influence graph of $P$, denoted by $R I G(P)$, has an edge between two distinct vertex-points $u, v \in P$ iff the open rectangle of influence of $u$ and $v$ is empty, i.e. $R(u, v) \cap P=\emptyset$. If $P$ contains three or more collinear points lying on a horizontal or a vertical line, then among those points, $R I G(P)$ only contains edges between the consecutive points of $P$ on the horizontal or vertical line. Clearly, $R I G(P)$ is an open RID graph, and an open rectangle of influence drawing of $R I G(P)$ can be obtained by connecting with straight-line segments the points of $P$ that correspond to pairs of adjacent vertices in $R I G(P)$. To simplify notation, in what follows $R I G(P)$ denotes both the open rectangle of influence graph of $P$ and its open rectangle of influence drawing with vertex-point set $P$.

The closed rectangle of influence graph of $P[13]$, denoted by $R I G[P]$ is defined similarly. It has an edge between two distinct vertex-points $u, v \in P$ iff $R[u, v] \cap P-\{u, v\}=\emptyset$. It is easy to see that $R I G[P]$ is a closed RID graph, and a drawing of $R I G[P]$ (also denoted $R I G[P]$ ) can be obtained as above.

A minimum spanning tree of $P$, denoted $M S T(P)$, is a spanning tree of $P$ such that the sum of the lengths of the edge-segments is minimum. In general, a set $P$ may have many minimum spanning trees (for example, $P$ might consist of the vertices of a regular polygon). Again, $M S T(P)$ denotes both the tree and a drawing of it.

The Gabriel graph of $P$, denoted $G G(P)$, is the graph that has an edge between two distinct vertex-points $u, v \in P$ iff $d^{2}(u, v)<d^{2}(u, w)+$ 
$d^{2}(v, w)$, for all $w \in P, w \neq u, v$. More geometrically, $u, v$ are adjacent iff the closed disk having $u, v$ as antipodal points does not contain any other vertexpoint except $u, v . G G(P)$ is connected and planar [19]. $G G(P)$ denotes the graph and its drawing with vertex-point set $P$.

Lemma 1. Let $P$ be a set of distinct points of the plane. Then

$$
M S T(P) \subseteq G G(P) \subseteq R I G[P] \subseteq R I G(P)
$$

One consequence of the above lemma is that both open and closed rectangle of influence drawable graphs are connected. A second consequence is stated in the following corollary.

Corollary 2. Let $P$ be a set of points such that $R I G(P)$ is a tree. Then

$$
M S T(P)=G G(P)=R I G[P]=R I G(P),
$$

and the minimum spanning tree of $P$ is unique.

\section{Properties of Open and Closed RID graphs}

We first give a geometric property of open and closed rectangle of influence drawings; second, based on this property, we present a theorem that allows us to define (open or closed) RID subgraphs of (open or closed) RID graphs.

Lemma 3. Let $G$ be an open (closed) $R I D$ graph, and let $\Gamma$ be an open (closed) rectangle of influence drawing of $G$. Let $u, v$ be two non-adjacent vertex-points of $\Gamma$ and let $P$ be the set of vertex points in $R(u, v)(R[u, v])$. Then there is a path $\Pi$ in $\Gamma$ between $u$ and $v$ such that all the vertex-points of $\Pi$ belong to $P$.

Corollary 4. Let $r$ be a horizontal (vertical) line, and let $P_{1} \subseteq P$ be the subset of points lying in one of the two halfspaces defined by $r$ and such that: (i) $\left|P_{1}\right| \geq 2$; (ii) no points of $P_{1}$ belong to $r$. Let $x, y$ be any pair of points of $P_{1}$. Then either $R I G(P)$ (RIG[P]) has the edge-segment $(x, y)$, or $R I G(P)(R I G[P])$ contains a path $\Pi$ from $x$ to $y$ such that all the vertex-points of $\Pi$ belong to $P_{1}$.

Theorem 5. Let $G$ be open (closed) RID. Suppose the set of vertices of $G$ can be partioned into three subsets $A, B$ and $K$ (where $A$ or $B$ may be empty), such that $K$ is a clique and there are no edges from $A$ to $B$. Then the subgraphs induced by $A \cup K, B \cup K$ and $K$ are also open (closed) $R I D$.

Proof. Let $\Gamma$ be an open (closed) rectangle of influence drawing of $G$. Without loss of generality assume that $A$ is non-empty. Suppose the deletion of the vertexpoints representing $A$ in $\Gamma$ creates a new edge-segment $(x, y)$. Then $R(x, y)$ $(R[x, y])$ contains only vertices from $A(A \cup\{x, y\})$. At least one of the vertices of $A$ is in the rectangle $R(x, y)(R[x, y])$ and $x$ and $y$ are not in $A$. By Lemma 3 , there is a path from $x$ to $y$ containing only vertices from $A$. So neither $x$ nor $y$ can be a vertex-point representing a vertex of $B$, by the definition of $B$. It follows that $x, y \in K$. Therefore $(x, y)$ is not a new edge-segment, because $K$ is a clique. 
In other words, if $K$ is a clique in an open or closed RID graph $G$, and if $A$ is such that all edges with an endpoint in $A$ have the other endpoint in $K \cup A$, then $A$ can be removed from $G$ and the resulting graph is still open or closed RID. This implies that the result of removing any vertex whose neighbors induce a clique is again RID. Also, if an open or closed RID graph $G$ has several biconnected components, then each of these components is open or closed RID.

\section{Classes of Open RID Graphs}

\subsection{Wheels}

A wheel $W_{n}, n \geq 4$, consists of a cycle $C_{n-1}$ together with a center vertex adjacent to all the cycle vertices.

Theorem 6. Every wheel $W_{n}$ with $n$ vertices is an open RID graph. Furthermore, an open rectangle of influence drawing of $W_{n}$ can be constructed in $O(n)$ time.

\subsection{Triangle-free Graphs}

In this section we first characterize triangle-free open RID graphs. Second we show that if a planar open RID graph admits an open rectangle of influence drawing $\Gamma$ where no two edge-segments cross, then all the internal faces of $\Gamma$ are 3 -cycles.

Theorem 7. A triangle-free graph is open RID iff is a simple path. Furthermore, an open rectangle of influence drawing of a triangle-free open RID graph with $n$ vertices can be constructed in $O(n)$ time.

Proof. We prove first that if a graph $G$ is open RID and contains no 3-cycles, then $G$ is a simple path. Let $\Gamma$ denote an open rectangle of influence drawing of $G$, and let $P$ be the set of vertex-points of $\Gamma$. First, suppose that some edge $(u, v)$ of $G$ is represented in $\Gamma$ by a horizontal or vertical edge-segment. Suppose $P$ contains a point not on the line $L$ through this segment. Let $p$ be a point in $P$ whose distance to $L$ is minimum. Then $R(p, v)$ and $R(p, u)$ are empty and $p, u, v$ is a 3-cycle in $G$, a contradiction. Hence all points of $P$ lie on the line $L$, and $G$ is a simple path.

Second, suppose that no edge of $G$ is represented in $\Gamma$ by a horizontal or vertical edge-segment. Consequently, no horizontal or vertical line can contain more than one vertex-point of $P$. Let $(u, v)$ be an edge-segment of $\Gamma$, and consider where the other points of $P$ can lie relative to the horizontal and vertical lines through $u$ and $v$. Without loss of generality, assume that both the $x$ - and $y-$ coordinates of $v$ are strictly greater than the corresponding coordinates of $u$. Clearly $R(u, v)$ is empty. Let $P^{\prime}$ denote the subset of $P$ consisting of points with $x$ - coordinate smaller than that of $v$ and $y$-coordinate larger than that of $u$. If $P^{\prime}$ is not empty, then any point $p^{\prime} \in P^{\prime}$ whose distance to $R(u, v)$ is minimum 
determines a 3 -cycle with $u$ and $v$ in $G$, a contradiction. Hence $P^{\prime}$ is empty. Similarly, the set $P^{\prime \prime}$ of points with $x$-coordinate larger than that of $v$ and $y$ coordinate smaller than that of $u$ is empty.

By the argument above, any vertex-points $w_{i}$ adjacent to $u$ and distinct from $v$ must be points whose $x$ - and $y$-coordinates are less than those of $u$; otherwise the rectangles $R\left(w_{i}, u\right)$ would contain $v$, which is impossible. Let $W$ denote the set $\left\{w_{i}\right\}$ of such vertex-points. We claim that $|W| \leq 1$. Let $w_{1}$ denote the vertex-point in $W$ with the largest $x$-coordinate. Then by the above argument with $u$ and $v$ replaced by $w_{1}$ and $u$, respectively, the remaining adjacent vertexpoints must have $x-$ and $y$-coordinates smaller than those of $w_{1}$. Hence if $W$ contains a second vertex-point $w_{2}$, the interior of $R\left(w_{2}, u\right)$ contains $w_{1}$, which is impossible. Hence $|W| \leq 1$. But $u$ represents any vertex of degree at least 1 in $G$. It follows that $G$ is a path.

The proof is completed by observing that any path $\Pi$ with $n$ vertices is open RID. Namely, an open rectangle of influence drawing of $\Pi$ can be produced by representing the vertices of $\Pi$ with $n$ collinear vertex-points. Clearly, this procedure requires $O(n)$ time.

Corollary 8. A bipartite graph is open RID iff it is a simple path.

Corollary 9. A tree is open RID iff it is a simple path.

Corollary 10. No cycles $C_{k}$ such that $k \geq 4$ are open $R I D$.

It is worth noticing that $C_{3}$ is open $\mathrm{RID}$; for example, an open rectangle of influence drawing of $C_{3}$ can be obtained by representing the vertices with vertex-points $(0,0),(0,2)$ and $(1,1)$.

In the rest of this subsection, we study whether a planar graph with some faces that are 3 -cycles can have a planar open rectangle of influence drawing, i.e. one such that no two edge-segments intersect except at common endpoints.

Lemma 11. Let $\Gamma$ be a planar open rectangle of influence drawing of an open RID graph. Then all internal faces of $\Gamma$ are 3-cycles.

Theorem 12. If a planar graph $G$ admits an open planar rectangle of influence drawing, then $G$ has a planar embedding where all internal faces are 3-cycles.

The converse of the above theorem does not hold. Consider for example the graph obtained by inserting a vertex in each of the internal faces of a wheel whose center has degree 4 and then adding edges so that all internal faces are triangulated. It is easy to see that such a graph does not have a planar open rectangle of influence drawing.

\subsection{Outerplanar Graphs}

We first characterize maximal outerplanar open RID graphs. Then we show that biconnected non-maximal outerplanar graphs are not open RID. 
Lemma 13. A maximal outerplanar graph $G$ with $n$ vertices such that its dual is a simple path is open RID. Furthermore, an open RID drawing of $G$ can be constructed in $O(n)$ time.

Proof. If $G$ has fewer than 5 vertices, a drawing can easily be found (for example if $G$ has four vertices, three of them can be represented as horizontally collinear and the fourth one as vertically collinear with one of the first three), so assume that $G$ has at least 5 vertices.

Observe first that $G$ has two vertices of degree 2. Label these vertices left and right. Divide the remaining vertices into two chains from left to right. Let $a_{1}$ and $a_{2}$ be neighbors of left and right, respectively, on one of the chains. Similarly, let $b_{1}$ and $b_{2}$ be the neighbors of left and right respectively on the second chain. Note that either $a_{2}$ or $b_{2}$ has degree 3 , but not both. Without loss of generality assume that $b_{2}$ has degree 3 . Order the chordal edges, i.e. edges that connect vertices from one chain to the other chain, compatibly with the order in which they are encountered as $G$ is traversed from left to right on each chain. So $\left(a_{1}, b_{1}\right)$ is the first chordal edge, $\left(a_{2}, b_{2}\right)$ is the last one.

An open rectangle of influence drawing of $G$ can be constructed as follows. Draw two non-parallel, non-intersecting line segments, one with a positive slope, and below it one with a negative slope. Place left on the top line segment, place vertex $a_{1}$ to its right. Place the remaining vertices of this chain in the order in which they appear on the chain, to the right of $a_{1}$. Place the vertices of the second chain on the bottom line segment, such that $b_{1}$ is to the right of left and the remaining vertices of the second chain are placed to the right of $b_{1}$ in the order in which they appear on the chain. Moreover no two vertices should lie on the same vertical line and the last chord of each vertex from either chain (except $b_{2}$ ) should be to the right of the vertical line through this vertex. Place the right vertex to the right of $b_{2}$ on the top line segment.

We can now verify that $R(u, v)$ is empty if and only if $(u, v)$ is an edge of $G$. The proof is concluded by observing that the time complexity of the algorithm implicit in this proof is $O(n)$.

Lemma 14. A maximal outerplanar graph whose dual consists of a vertex of degree 3 adjacent to three vertices of degree 1 is not open RID.

The result of the previous lemma can be generalized as follows.

Lemma 15. Any maximal outerplanar graph whose dual tree has a vertex of degree 3 is not open RID.

Lemma 16. Any biconnected component of an outerplanar open RID graph is maximal outerplanar.

The following theorem summarizes the results of this section.

Theorem 17. A biconnected outerplanar graph $G$ with $n$ vertices is an open $R I D$ graph iff it is maximal outerplanar and its dual is a path. If $G$ is open RID, an open rectangle of influence drawing of $G$ can be constructed in $O(n)$ time. 


\section{Classes of Closed RID Graphs}

\subsection{Wheels and Cycles}

Theorem 18. Every wheel $W_{n}$ with $n$ vertices is closed RID. Furthermore, a closed rectangle of influence drawing of $W_{n}$ can be constructed in $O(n)$ time.

Note that it is not possible to construct a planar closed RID rectangle of influence drawing of $K_{4}$, since this would require having a vertex-point inside a triangle and outside the rectangle of influence of any of the edge-segments in the triangle, which is impossible.

Theorem 19. Every cycle $C_{n}$ is a closed RID graph. Furthermore, a closed rectangle of influence drawing of $C_{n}$ can be constructed in $O(n)$ time.

\subsection{Trees}

The following Lemma is a consequence of Corollary 2 and of [2], where it is proved that given a set $P$ of points such that $G G(P)$ is a tree, then the angle between any two incident edges is greater or equal to $\pi / 2$.

Lemma 20. Let $P$ be a set of points such that $R I G(P)$ is a tree. Then the angle between any two consecutive edges is greater or equal to $\pi / 2$.

Corollary 21. A closed RID tree has vertices with degree at most 4 .

Lemma 20 together with Corollary 4 have important implications for the shape of the closed rectangle of influence drawing of a tree, as stated in the following lemma.

Lemma 22. Let $T$ be a closed RID tree, let $\Gamma$ be a closed rectangle of influence drawing of $T$, and let $x$ be a vertex-point of $\Gamma$.

1. If $\operatorname{deg}(x)=3$ at least two of the edge-segments incident on $x$ are axis-aligned.

2. If $\operatorname{deg}(x)=4$ all edge-segments incident on $x$ are axis-aligned.

Theorem 23. A tree $T$ with $n$ vertices is a closed RID tree iff its vertices have maximum degree at most 4 and it has at most four leaves.

Furthermore, if $T$ is closed RID, a closed rectangle of influence drawing of $T$ can be constructed $O(n)$ time.

\subsection{Maximal Outerplanar Graphs}

We give here a partial characterization.

Lemma 24. Any maximal outerplanar graph $G$ with $n$ vertices whose dual is a tree with at most 1 vertex of degree 3 is closed RID. Furthermore, a closed rectangle of influence drawing of $G$ can be constructed in $O(n)$ time. 
Lemma 25. No maximal outerplanar graph whose dual has at least 3 vertices of degree 3 is closed RID.

Lemma 26. At least some maximal outerplanar graphs whose dual tree has two vertices of degree 3 are closed RID.

The results of this section are summarized in the following theorem.

Theorem 27. Every maximal outerplanar graph whose dual has at most one vertex of degree 3 is a closed RID graph. No maximal outerplanar graph whose dual has at least three vertices of degree 3 is a closed RID graph. There exist maximal outerplanar closed RID graphs whose dual has two vertices of degree 2 .

\section{Open Problems}

Several problems remain open in this area. We mention here two that we consider particularly interesting.

1. Give a complete characterization of (maximal) outerplanar closed RID graphs.

2. Study other families of open and closed rectangle of influence drawable graphs. For example, it would be interesting to know which types of planar triangulated graphs have a (planar) open or closed rectangle of influence drawing.

\section{Acknowledgments}

Most of the results of this paper have been obtained while four of the authors were participating the International Workshop on Geometric and Visibility Representations of Graphs, co-organized by D. Rappaport and S. Whitesides and held at the Bellairs Research Institute of McGill University, Feb. 11-17, 1994. We are grateful to the other participants of the Workshop for many useful discussions.

\section{References}

1. J. A. Bondy and U. S. R. Murty. Graph Theory with Applications. Elsevier Science, New York, New York, 1976.

2. P. Bose, W. Lenhart, and G. Liotta. Characterizing Proximity Trees. Algorithmica: Special Issue on Graph Drawing (to appear). Also available as TR-SOCS 93.9, School of Computer Science, McGill Univ., 1993.

3. P. Bose, G. Di Battista, W. Lenhart, and G. Liotta. Proximity Constraints and Representable Trees. Proc. GD'94, 1994.

4. R. J. Cimikowski. Properties of Some Euclidean Proximity Graphs. Patt. Recogn. Letters, 13, 1992, pp. 417-423. 
5. M. de Berg, S. Carlsson, and M.H. Overmars. A General Approach to Dominance in the Plane. J. of Algorithms, 13, 1992, pp. 274-296.

6. G. Di Battista, P. Eades, R. Tamassia and I.G. Tollis. Algorithms for Automatic Graph Drawing: An Annotated Bibliography. Computational Geometry: Theory and Applications (to appear).

7. G. Di Battista, W. Lenhart, G. Liotta. Proximity Drawability: a Survey. Proc. GD'94, 1994.

8. G. Di Battista and L. Vismara. Angles of Planar Triangular Graphs. Proc. STOC '93, 1993, pp. 431-437.

9. M. B. Dillencourt and W. D. Smith. Graph-Theoretical Conditions for Inscribability and Delaunay Realizability. Proc. CCCG'94, 1994, pp.287-292.

10. P. Eades and S. Whitesides. The Realization Problem for Euclidean Minimum Spanning Tree is NP-hard. Proc. ACM Symp. on Comp. Geom., pp. 49-56, 1994.

11. H. de Fraysseix, J. Pach, and R. Pollack. Small Sets Supporting Fary Embeddings of Planar Graphs. Proc. STOC '88, 1988, pp. 426-433.

12. H. de Fraysseix, J. Pach, and R. Pollack. How to Draw a Planar Graph on a Grid. Combinatorica, 10, 1990, pp. 41-51.

13. M. Ichino, J. Sklansky. The Relative Neighborhood Graph for Mixed Feature Variables. Patt. Recogn., 18, 1985, pp. 161-167.

14. J. W. Jaromczyk and G. T. Toussaint. Relative Neighborhood Graphs and Their Relatives. Proc. of the IEEE, 80, 1992, pp. 1502-1517.

15. G. Kant. Drawing Planar Graphs Using the lmc-ordering. Proc. FOCS '92, 1992, pp. 101-110.

16. D. G. Kirkpatrick and J. D. Radke. A Framework for Computational Morphology. Computational Geometry, ed. G. T. Toussaint, Elsevier, Amsterdam, 1985, pp. 217248.

17. P. M. Lankford. Regionalization: Theory and Alternative Algorithms. Geogr. Anal., 1, 1969, pp. 196-212.

18. A. Lubiw and N. Sleumer. All Maximal Outerplanar Graphs are Relative Neighborhood Graphs. Proc. CCCG '93, 1993, pp. 198-203.

19. D. W. Matula and R. R. Sokal. Properties of Gabriel Graphs Relevant to Geographic Variation Research and the Clustering of Points in the Plane. Geogr. Anal., 12, 1980, pp. 205-222.

20. M. H. Overmars and D. Wood. On Rectangular Visibility. Journal of Algorithms, 9, 1988, pp. 372-390.

21. M. S. Paterson and F.F. Yao, On Nearest-Neighbor Graphs. Proc. ICALP '92, 1992, pp. 416-426.

22. F. P. Preparata and M. I. Shamos, Computational Geometry: an Introduction. Springer-Verlag, New York, 1985.

23. J. D. Radke. On the Shape of a Set of Points. Computational Morphology, ed. G. T. Toussaint, Elsevier, Amsterdam, 1988, pp. 105-136.

24. G. T. Toussaint. The Relative Neighborhood Graph of a Finite Planar Set. Patt. Recogn., 12, 1980, pp. 261-268. 